\title{
Le Programme d'Agriculture Urbaine de la ville de Rosario en Argentine
}

Une gestion complexe de la multifonctionnalité

La compleja gestión de la multifuncionalidad en el Programa de Agricultura

Urbana de la ciudad de Rosario en Argentina

The complex management of multifunctionality in the Urban Agriculture

Programme in the city of Rosario, Argentina

\section{Marta Soler Montiel}

\section{OpenEdition} Journals

Édition électronique

URL : http://journals.openedition.org/ethnoecologie/2390

DOI : $10.4000 /$ ethnoecologie. 2390

ISSN : 2267-2419

\section{Éditeur}

Laboratoire Eco-anthropologie et Ethnobiologie

\section{Référence électronique}

Marta Soler Montiel, « Le Programme d'Agriculture Urbaine de la ville de Rosario en Argentine », Revue d'ethnoécologie [En ligne], 8 | 2015, mis en ligne le 31 décembre 2015, consulté le 01 mai 2019. URL : http://journals.openedition.org/ethnoecologie/2390; DOI : 10.4000/ethnoecologie.2390

Ce document a été généré automatiquement le 1 mai 2019.

\section{(i)}

Revue d'ethnoécologie est mis à disposition selon les termes de la licence Creative Commons Attribution - Pas d'Utilisation Commerciale - Pas de Modification 4.0 International. 


\title{
Le Programme d'Agriculture Urbaine de la ville de Rosario en Argentine
}

\author{
Une gestion complexe de la multifonctionnalité
}

La compleja gestión de la multifuncionalidad en el Programa de Agricultura

Urbana de la ciudad de Rosario en Argentina

The complex management of multifunctionality in the Urban Agriculture

Programme in the city of Rosario, Argentina

\section{Marta Soler Montiel}

\section{Introduction}

1 Par définition, la principale caractéristique de l'agriculture urbaine est d'être intégrée dans le système économique et écologique urbain (Mougeot 2000). L'incorporation de l'agriculture dans l'espace de la ville suppose de reconnaître la multifonctionnalité tant du sol urbain que de l'agriculture, car au-delà de la production de vivres, les potagers sont susceptibles de générer d'amples bénéfices socioéconomiques et écologiques (Renting et al. 2013, CBD 2012, Zasada 2011) dans les lieux où ils s'implantent. En période de crise économique notamment, l'agriculture urbaine se voit dotée de nombreuses qualités. La principale semble résider dans sa capacité à nourrir directement la population et à constituer une source d'emplois et de revenus (FAO 2014, Banque mondiale 2013). Quand les pratiques culturales sont " écologiques ", les aliments qu'elle fournit à la population urbaine sont, en outre, sains et de qualité. Dans le même temps la pratique de l'agriculture urbaine est vantée pour ses bienfaits sur le corps et l'esprit (Bellows et al. 2005, Largo-Wight 2011) pour ses effets positifs en matière de convivialité, sans compter qu'elle génère des possibilités de loisirs et favorise l'éducation à l'environnement pour de larges pans de la population urbaine (Zasada 2011). 
Figure 1 : Maraîchère du Programme d'Agriculture Urbaine avec ses enfants dans le parc-jardin Molino blanco de Rosario (Argentine)

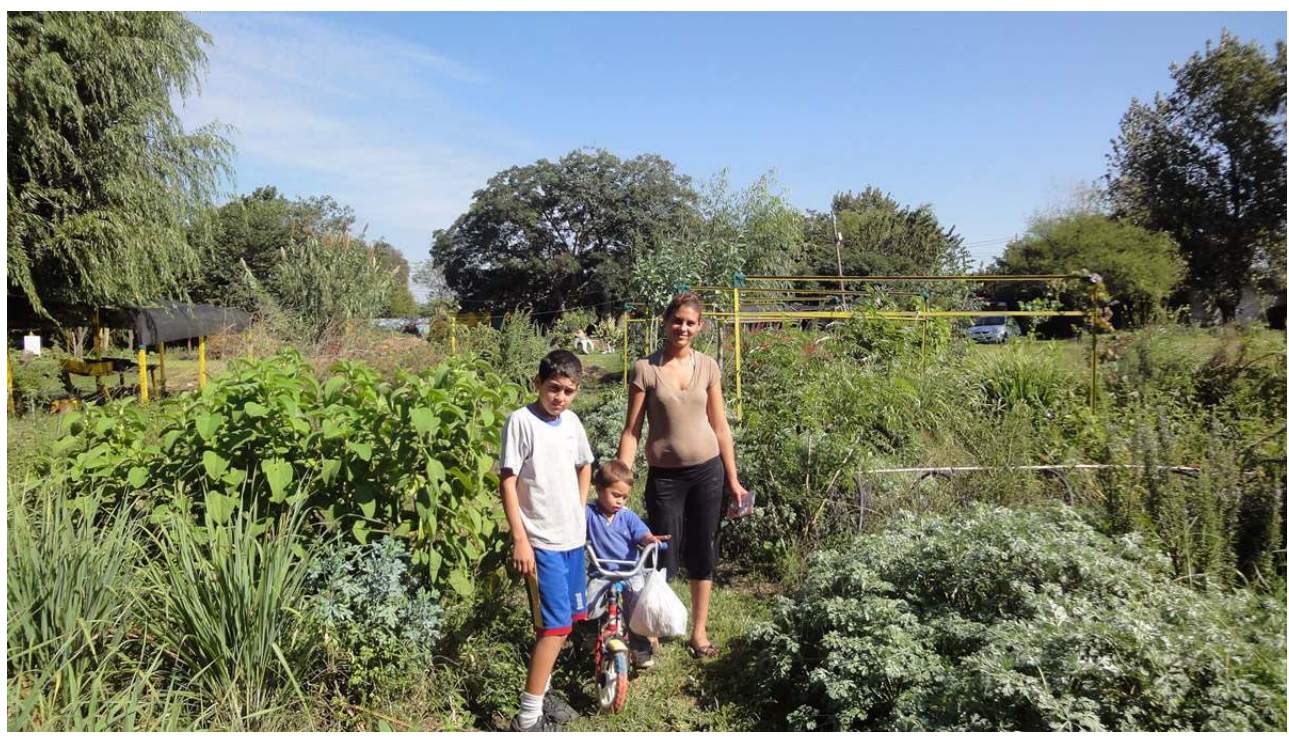

(C) Programa de Agricultura Urbana de Rosario, Argentina

2 D'un point de vue écologique, l'intégration dans la ville de l'agriculture urbaine et périurbaine génère des services environnementaux et écosystémiques. Il en va ainsi des bénéfices apportés par le « reverdissement » des villes, la création de microclimats, d'une meilleure protection de la biodiversité (CBD 2012), des économies d'énergie à travers la réduction des distances entre production et consommation d'aliments, ou de la réutilisation des déchets organiques. Plus récemment, la contribution de l'agriculture urbaine à la création de villes résilientes, comme frein au changement climatique, a été soulignée (De Zeeuw 2011).

3 Nous partons donc ici de l'idée, largement documentée, que cette agriculture est multifonctionnelle et qu'elle a démontré sa capacité à répondre aux enjeux de la crise globale actuelle, en apportant des revenus à ceux qui travaillent la terre, des aliments sains et durables à ceux qui mangent ses produits, ainsi que divers services environnementaux et sociaux aux citoyens dans leur ensemble. Nous avons ainsi choisi de creuser plus particulièrement la question de la gestion et la planification de cette multifonctionnalité et de son intégration dans les politiques de la ville, car elles sont moins étudiées et sujettes à débat. 
Figure 2 : Panneau à l'entrée du parc-jardin Molino blanco, construit sur un ancien dépôt à ordure d'un bidonville de Rosario

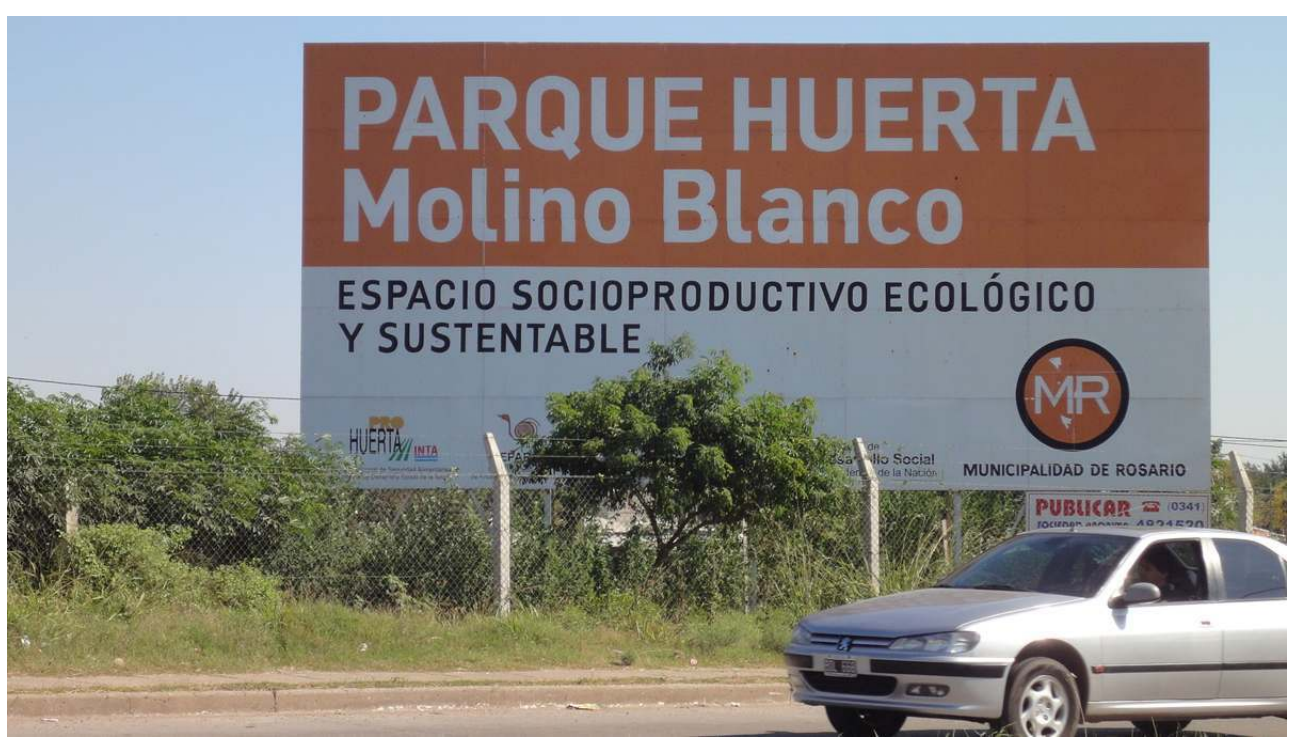

CLICHÉ M. SOLER MONTIEL

4 Nous le ferons au travers de l'analyse du Programme d'Agriculture Urbaine (PAU) de la ville de Rosario, qui offre un bon exemple de complexité et de contradictions. L'analyse se fonde sur un travail de terrain réalisé en avril et août 2010. L'enquête s'est appuyée sur des visites de jardins potagers et de marchés du Programme, sur des entretiens avec des maraîchers et des maraîchères, sur la participation à diverses activités proposées par le PAU. Un atelier a été organisé afin d'élaborer un diagnostic de ce programme ( $\left.\mathrm{DAFO}^{1} »\right)$. Quinze entretiens approfondis ont été menés auprès des membres de l'équipe technique, de l'Université Nationale de Rosario et de la Municipalité. En 2014, les données recueillies ont été actualisées grâce à des informateurs locaux et à la bibliographie disponible.

\section{Complexification}

5 Rosario compte un million d'habitants, c'est la troisième ville d'Argentine et (INDEC 2010). Établie au bord du fleuve Paraná, dans la province de Santa Fe au centre-est du pays, elle est née et s'est développée avec l'industrie, et elle a reçu d'importants flux migratoires étrangers et nationaux, en particulier des zones rurales du nord du pays.

6 C'est également un pôle économique, financier et culturel important. Elle se trouve au cœur de la Pampa humide, l'une des principales zones de production de soja. Son port est le principal centre d'exportation de la production céréalière du pays, et il héberge l'un des plus grands complexes de traitement du soja au monde. Cette forte polarisation économique est rendue visible par la ceinture de bidonvilles qui entoure, aujourd'hui, encore, le centre urbain. C'est là que vit la population la plus précaire et la plus marginalisée, dont une partie importante est issue des migrations venues des zones rurales appauvries, spécialement du nord du pays. L'autre provient des populations exclues du marché du travail industriel, en raison des processus de restructuration économique induits par la mondialisation dans les trente dernières années². 
7 C'est dans ce contexte que le Programme d'Agriculture Urbaine de Rosario est né voilà une trentaine d'années. Trois grandes étapes peuvent être identifiées.

- 1987-1990 : définition d'une méthodologie agroécologique pour l'agriculture urbaine

À la fin des années 1980, un groupe de techniciens, avec une forte représentation d'agronomes, se joint à des membres de la Coopérative Saladillo localisée sur la commune d'El Mangrull ${ }^{3}$. Ils proposent la création de jardins communautaires en ville, dans l'esprit d'une démarche participative d'intervention sociale à destination des populations pauvres. L'objectif est de sortir de l'assistanat installé par les politiques de distribution d'aliments. Il faut s'abstraire du cercle vicieux de l'exclusion dans lequel les gens, piégés par le nécessité de résoudre leurs difficultés quotidiennes, perdent toute capacité à planifier et à développer leurs propres projets de vie (Lattuca 2006, Mazzuca et al. 2009). Plus tard ce groupe deviendra le Centre d'études pour la production agroécologique de Rosario (CEPAR). La démarche trouve ses fondements théoriques et méthodologiques en agroécologie, dans le cadre de l'Institut de Sociologie et d'études paysannes (ISEC) de l'Université de Cordoue (Espagne)4. Partant de la tradition des études paysannes (Sevilla Guzmán 2006), l'ISEC propose une stratégie d'intervention à trois niveaux : technico-productif, socio-économique et culturel, mais aussi politique. L'objectif final est de favoriser la « repaysannisation » de la société (Pérez-Vitoria 2005, Ploeg 2008). La dimension technico-productive de l'agroécologie redessine les agroécosystèmes avec des critères écologiques pour assurer une alimentation suffisante et acquérir une autonomie par rapport au marché des intrants (Altieri 1987, Gliessman 1989). La dimension socioéconomique et culturelle cherche à promouvoir des stratégies d'organisation et d'articulation avec les marchés en partant des valeurs traditionnelles paysannes. Autrement dit, il s'agit de proposer une stratégie de « repaysannisation » culturelle et matérielle (Toledo 1993, Sevilla Guzmán 2006, Ploeg 2008). La dimension politique vise à transformer les rapports de pouvoir en faveur des exclu(e)s du système agroalimentaire (Sevilla Guzmán 2006).

Dans ces années-là, l'agriculture urbaine de Rosario se développe en marge de tout soutien institutionnel, ce qui ralentit le processus. Mais cela a permis un apprentissage collectif et l'émergence d'une méthodologie participative et concrète, car appliquée à la création de jardins communautaires.

- 1990-2001 : transition et première institutionnalisation

Après la crise provoquée par l'hyper inflation des années 1980, les institutions publiques ont cherché à mettre en place des politiques d'intervention sociale et économique. La municipalité de Rosario, en lien avec d'autres institutions et $\mathrm{ONG}^{5}$, institutionnalise un premier Programme de jardins communautaires qui fonctionne entre 1990 et $1997^{6}$. Entre 1998 et 2001, ce programme est démantelé et intégré dans le programme municipal CROÎTRE exclusivement orienté vers les enfants en situation de vulnérabilité sociale et vers la promotion de jardins familiaux destinés aux parents.

Ce changement a signifié l'échec de la stratégie des jardins communautaires proposée par le CEPAR, qui allait bien au-delà de la création de jardins familiaux d'autoconsommation auquel se cantonnait le programme CROÎTRE. Le CEPAR ne voulait pas se limiter au travail avec des familles nucléaires mais cherchait à élargir la démarche à toute la population dans le besoin, disposée à s'impliquer dans un projet collectif d'agriculture urbaine. Il avait de plus, l'ambition de promouvoir des formes d'organisation et de participation collectives afin de rompre avec l'individualisme qui règne dans la société. Ce premier échec du CEPAR a constitué un apprentissage des limites et des conditions factuelles du travail avec l'administration, dont les exigences politiques en termes de délais et de résultats sont déterminants pour obtenir la stabilité des programmes publics (Lattuca 2006). 
- Depuis 2002 : consolidation de l'agriculture urbaine en tant que politique municipale Depuis la nouvelle crise financière de 2001, l'agriculture urbaine de Rosario est devenue une activité massive, les habitants s'organisant pour satisfaire leurs besoins de base face à l'effondrement économique. Dans ce contexte d'urgence sociale, la municipalité a rapidement adopté la proposition du CEPAR de créer un Programme d'Agriculture Urbaine, en intégrant cette activité dans la politique municipale de lutte contre la crise. En 2002, le PAU s'institutionnalise par la signature d'une convention entre la Municipalité, le CEPAR et le Programme Pro-Huerta de l'Institut National de Technologies agricoles (INTA).

Entre 2002 et 2004, le PAU connaît une participation massive avec des jardins de plus de 100 personnes où se combinent les activités de production pour l'autoconsommation, l'autoconstruction de fours, la vente sur des marchés, accompagnées d'actions de formation et de conscientisation agroécologique qui ont permis des apprentissages coopératifs et solidaires (Mazzuca et al. 2009). Cette participation active de la population est liée à une politique publique d'indemnités de chômage. À l'époque, l'obtention de ces subsides était conditionnée par la réalisation d'une activité sociale, dont le travail communautaire du PAU faisait partie.

Ces années ont été créatives, la mise à disposition d'infrastructures permettant la création de nouveaux jardins, favorisant le développement des marchés et des agro-industries ${ }^{7}$. La municipalité réglemente alors l'agriculture dans la ville, ce qui lui donne une sécurité juridique, une reconnaissance publique et une légitimité politique. Le PAU gagne une visibilité et une reconnaissance internationales, comme le montre l'obtention du prix décerné à l'un de dix meilleurs projets de lutte contre la pauvreté dans le monde (prix accordé par l'ONU Habitat et la municipalité de Dubaï).

Figure 3 : Maraîchers arrosant leur potager dans le Parc-jardin Molino blanco du PAU

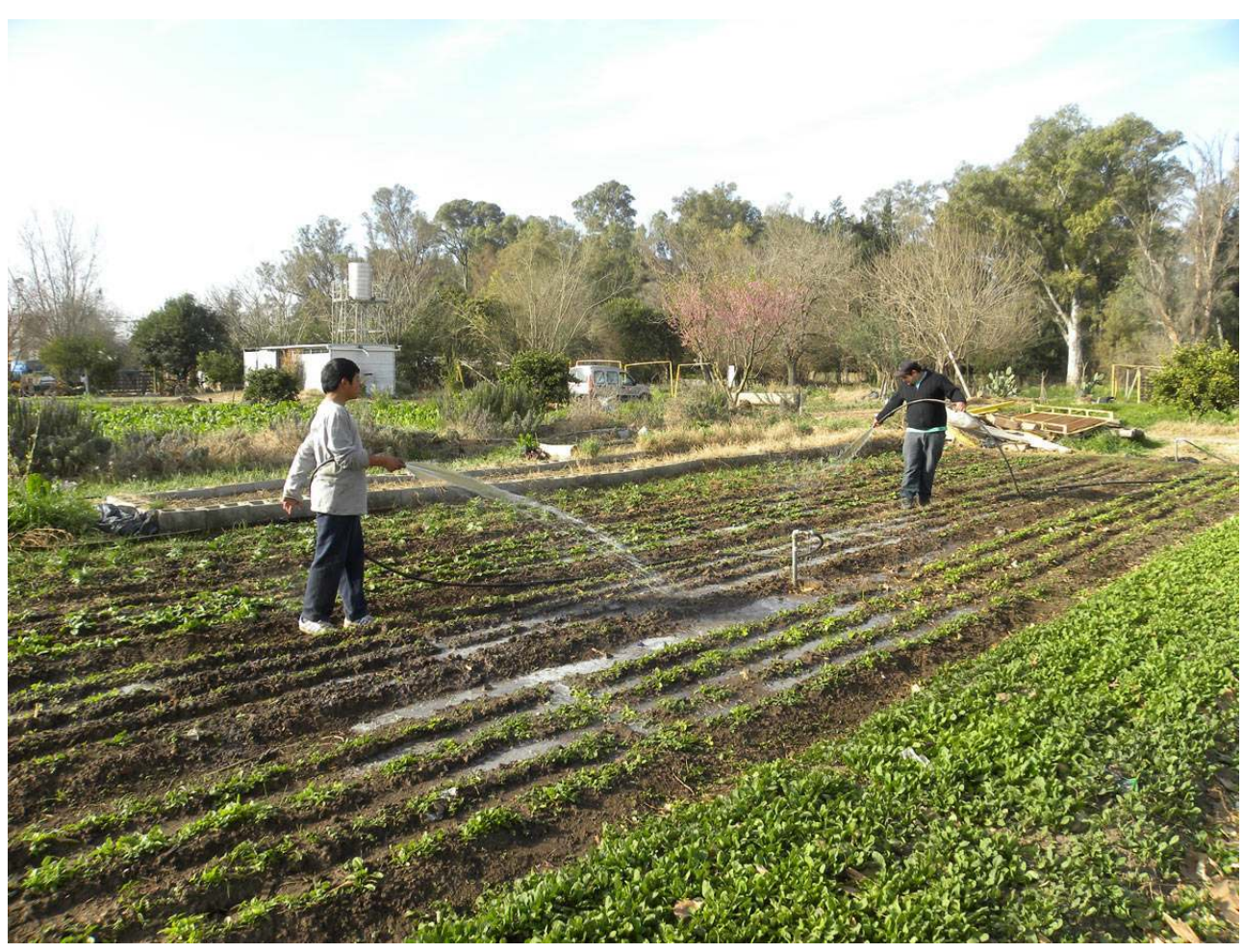

(c) Programa de Agricultura Urbana de Rosario, Argentina 
Entre 2005 et 2010, le PAU connaît une deuxième étape d'institutionnalisation, alors que la participation dans les jardins diminue du fait de l'amélioration de la conjoncture économique. Cette moindre implication n'est pas étrangère au fait qu'en 2005 l'indemnité chômage a été déconnectée de la nécessité de fournir une prestation sociale.

Pendant ces années, la réglementation est renforcée et la priorité est donnée à la stabilisation de l'occupation des sols. Les dotations en matériel et en infrastructures reçues par le Programme augmentent ${ }^{8}$. Depuis 2011, date d'apogée de la dotation en matériel et de la consolidation de l'organisation, le PAU vit une nouvelle étape de développement et de diversification de ses activités. Elle sera analysée en détail dans la troisième partie de cet article.

Dans ce processus de construction, de plus en plus de personnes s'impliquent. Les espaces concernés et les activités réalisées, l'organisation générale et les relations institutionnelles se complexifient. Aujourd'hui le PAU est un réseau de relations et d'agents multiples qui développent leurs activités sur divers terrains et lieux de la ville, connectant trois types d'espaces, institutionnel, agroalimentaire et citoyen (Figure 4). L'équipe technique assure l'articulation.

Figure 4 : Le réseau du Programme d'Agriculture Urbaine de la ville de Rosario, Argentine

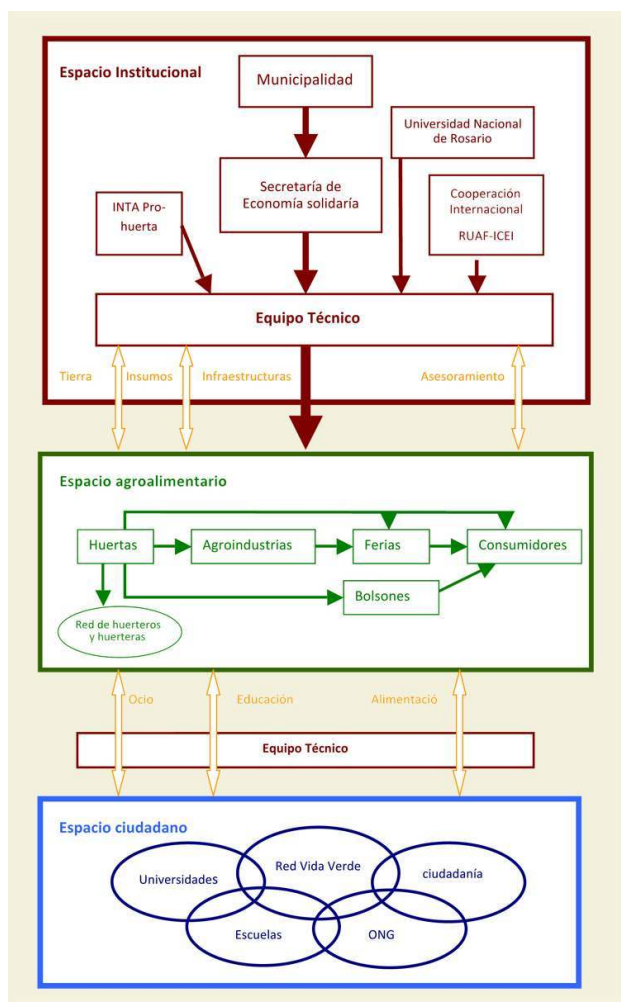

\section{La chaîne agroalimentaire urbaine du PAU}

9 Ce sont les maraîchères et les maraîchers qui forment le cœur du PAU. Ils sont structurés en réseau depuis 2005, qui les constitue en sujet collectif autonome9. Il est difficile de préciser le nombre de personnes actuellement engagées, en raison des nombreux mouvements d'entrée et de sortie et de l'absence de registres. Mais on estime que 150 à 200 personnes cultivent de manière stable dans les espaces gérés par le programme. Un 
nombre égal de personnes travaillerait dans les programmes de formation pour la professionnalisation de l'agriculture urbaine comme celui qui est intitulé « Más y mejor trabajo » (Plus de travail et meilleur travail), réalisé en collaboration avec le Ministère du travail et destiné aux jeunes de 18 à 25 ans.

Les maraîchers du PAU constituent un collectif hétérogène dont le point commun est la pauvreté. Un groupe important de personnes est d'origine rurale, en majorité issues du nord du pays, qui ont migré vers la ville en raison de la modernisation agricole qui a réduit l'emploi agricole et expulsé les petits paysans de leurs terres. C'est dans ce groupe que l'on trouve ceux qui font preuve des meilleures capacités de production, de l'investissement le plus fort dans les jardins. Les parcs-jardins ${ }^{10}$ Molino blanco et Hogar español sont cultivés par deux grandes familles, qui ont développé leur mode de vie autour de l'agriculture urbaine depuis trois générations. Les hommes et les femmes se répartissent le travail dans les jardins. Le rôle des femmes est primordial, ce sont elles qui assurent avec les revenus, les soins, l'alimentation et l'éducation des enfants et des petitsenfants.

11 Une grande partie des promoteurs du PAU ${ }^{11}$ sont des migrants du nord du pays qui conservent des savoirs paysans et indigènes en matière de pratiques culturales et des connaissances sur l'usage des fruits et légumes, des plantes aromatiques et des herbes médicinales.

Mais actuellement la majorité des maraîchers et maraîchères est d'origine urbaine, bien qu'ils descendent parfois de familles rurales. Nées en ville, ces personnes n'ont pas de lien antérieur ni d'expérience pratique en agriculture, ce qui rend difficile une intégration stable au programme. Néanmoins, quelques personnes d'origine urbaine, dans la majorité des cas issues des classes moyennes appauvries, sont devenues des maraîchers à plein temps avec une forte capacité de production et de travail. Par exemple, un ancien ouvrier de l'industrie de la viande de la ville, qui a perdu son emploi au moment de la crise de 2001, a transféré sa culture ouvrière dans le potager. Il cultive tous les matins entre 6 heures et midi, le même horaire que celui qu'il avait dans l'industrie, il commercialise sa production dans les différents circuits de vente et il obtient une des productions et un des revenus parmi les plus élevés du Programme. 
Figure 5 : Maraîchère du parc-jardin Molino blanco du PAU

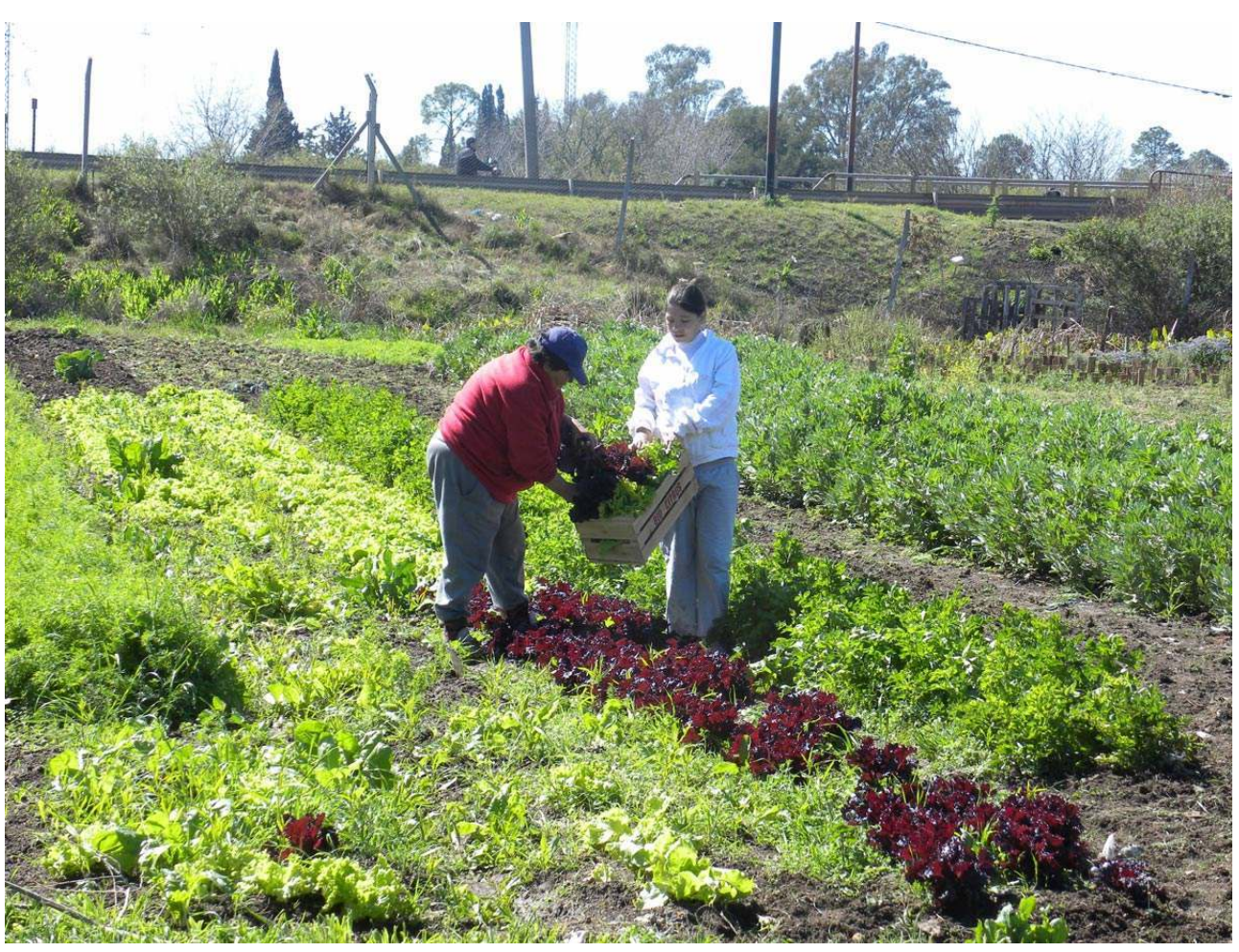

(C) Programa de Agricultura Urbana de Rosario, Argentina

13 Il faut souligner que $65 \%$ de ceux qui cultivent les jardins sont des femmes avec charges de famille, ce qui réduit leur possibilité de travailler à plein temps dans l'agriculture. Ces femmes, mais c'est aussi le cas de certains hommes, vivent souvent des situations sociales extrêmes, avec de la violence, de la drogue, des maternités précoces et un chômage chronique. Provenant majoritairement des bidonvilles de la périphérie de Rosario, les maraîchers du PAU ont vécu et vivent dans des conditions d'extrême précarité et d'exclusion sociale, ce qui se traduit par une grande fragilité psychologique, une faible estime de soi, des difficultés à s'engager de façon stable dans une activité, et des difficultés à entrer en relation avec des personnes en dehors de leur cercle proche. Ils ont du mal à être autonomes dans certaines tâches complexes comme la planification et la gestion des ventes.

La variété des situations sociales et personnelles de ceux qui cultivent au sein du PAU se traduit par des capacités de production et d'organisation différenciées, ce qui implique des formes de soutien technique et social diversifiés dans les multiples activités requises.

Les territoires productifs du PAU sont aussi très diversifiés, mais dans leur grande majorité il s'agit d'espaces intra-urbains vides sans qualité agronomique ${ }^{12}$, même si des pratiques écologiques continues ont permis de rendre ces sols cultivables. L'activité de production s'accomplit dans cinq parcs-jardins, cinq jardins communautaires agroécologiques et dix-sept potagers agroécologiques, deux systèmes de production organoponique $^{13}$, un espace de conservation de la biodiversité, une banque de semences a Ñanderoga et la pépinière municipale pour la production de semis et de biofertilisants. 
Figure 6 et 7 : Potagers urbains du PAU près des voies de chemin de fer de Rosario

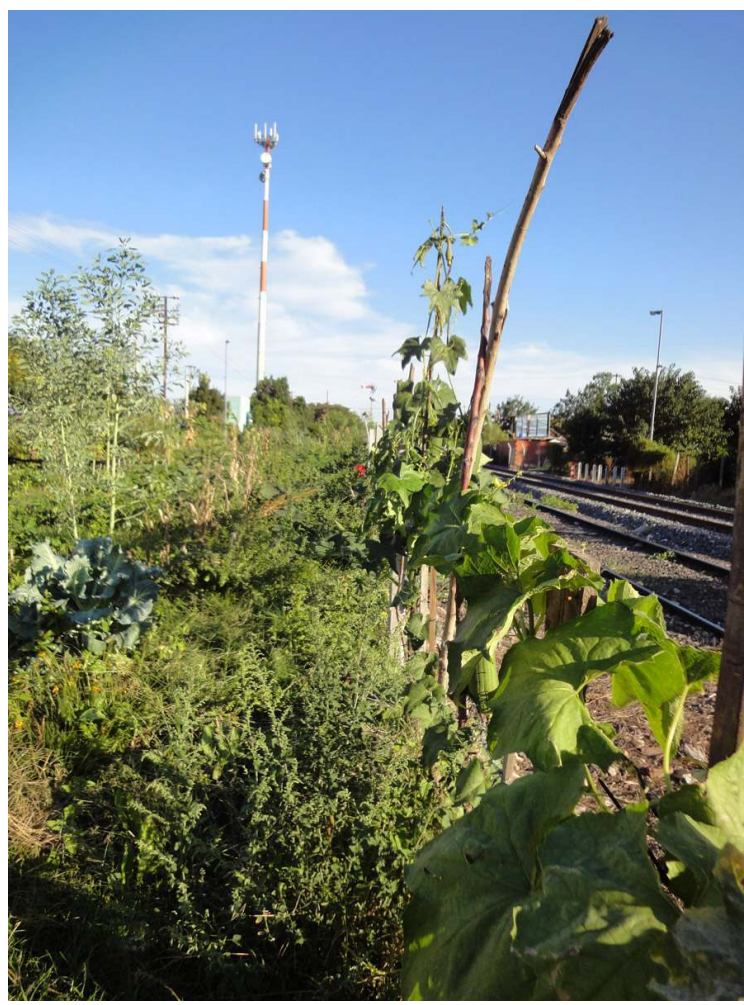

Cliché M. Soler Montiel

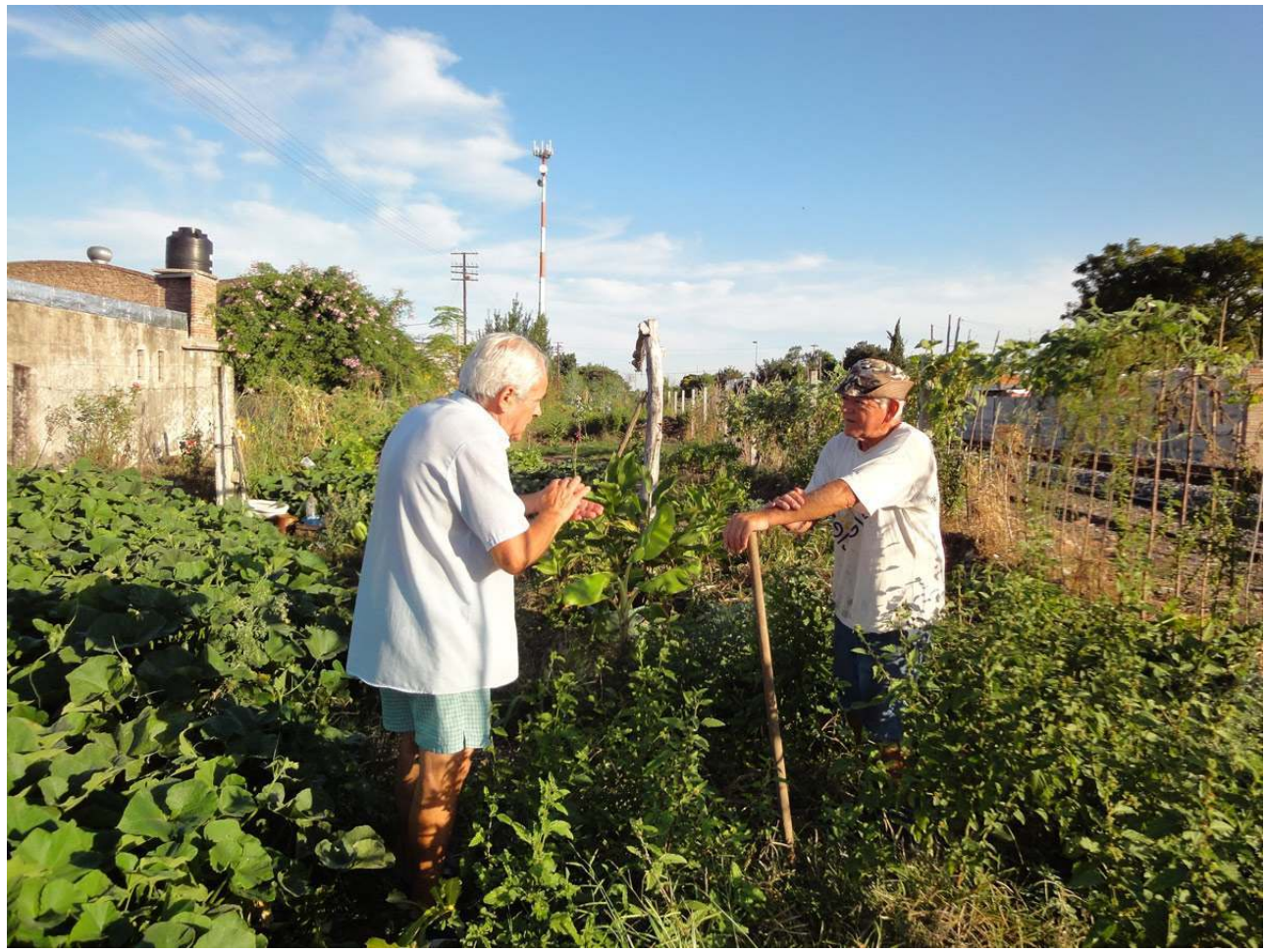

Cliché M. Soler Montiel

16 Les potagers communautaires et agroécologiques se situent dans différents lieux qui ont été progressivement intégrés au Programme à l'initiative des habitants, ou de l'équipe 
technique. Il peut s'agir de terrains proches de voies de chemin de fer et d'autoroutes, ou de terrains abandonnés réputés non constructibles ${ }^{14}$. Les parcs-jardins, dont le premier, Molino Blanco ${ }^{15}$, a été créé en 2005, sont en revanche des espaces planifiés, situés sur des terrains publics (il en est ainsi pour Tablada et Bosque de Constituyentes), ou cédés durablement par des institutions (la résidence de troisième âge Hogar Español par exemple). Les parcs-jardins sont donc conçus comme des espaces multifonctionnels ouverts à tous, pour des activités de loisirs et d'éducation en sus de la production agricole.

17 Dans les jardins du Pau on produit surtout des légumes, mais aussi des herbes aromatiques, des plantes médicinales et ornementales et dans une moindre mesure des fruits. Une partie des légumes est lavée, coupée, emballée pour être vendue. On prépare aussi des garnitures de tartes, des crèmes, des produits d'hygiène et des cosmétiques naturels dans deux "agro-industries", auxquelles les maraîchers peuvent vendre une partie de leur production.

Les fruits et légumes des jardins et les produits des agro-industries sont commercialisés dans un réseau de six marchés agroécologiques situés sur des places connues et emblématiques du centre-ville. On en trouve aussi dans certaines municipalités des arrondissements. Les marchés sont hebdomadaires et font partie d'un système relevant de l'économie solidaire de la municipalité. Le montage et le démontage des marchés, sont pris en charge par le Sous-secrétariat de l'Économie solidaire, intégré dans le Secrétariat de la Promotion sociale de la Municipalité, en collaboration avec les maraîchers.

La distribution hebdomadaire de paniers a commencé en 2010 et représente actuellement un nouveau circuit de commercialisation avec une distribution de 40 à 50 paniers chaque semaine à un réseau stable de consommateurs, ce qui rapporte à chaque personne un revenu moyen de 500 pesos $^{16}$, qui viennent compléter les revenus des marchés (RUAF 2013). La commercialisation de paniers demande une planification de la production et une coordination entre production et commercialisation, ce qui a permis de développer des capacités d'organisation collective (Sánchez Minarro 2014, Mazzuca et al. 2009). Les premières années, la vente et la distribution de paniers de légumes furent conçues, mises en place et coordonnées par l'équipe technique. Actuellement on est dans une phase de transition: le réseau de maraîchers gagne en autonomie de gestion. Une partie des légumes (en plus de la partie destinée à l'autoconsommation) est vendue directement et individuellement, soit sur place dans les jardins, soit dans les quartiers environnants.

\section{Le réseau institutionnel du Programme d'Agriculture Urbaine}

L'équipe technique constitue, à côté des maraîchères et maraîchers, l'agent principal du PAU, dans la mesure où ce sont ses membres qui interviennent, et qui souvent représentent le collectif des maraîchers, dans les relations institutionnelles et dans les rapports citoyens (à un degré moindre). Elle est actuellement composée de 25 membres, techniciens et promoteurs, et elle est dotée d'un budget annuel de 380000 dollars (FAO 2014).

21 La direction et les techniciens les plus anciens sont issus du CEPAR et sont liés au Programme depuis son origine. L'équipe technique s'est étoffée avec deux types de profils bien différenciés. Lors de la phase initiale de l'institutionnalisation du PAU, on a recruté une équipe de " promoteurs » sélectionnés parmi les plus actifs des maraîchers, ceux qui se montraient les plus dynamiques et soucieux du lien social, et qui sont devenus des 
employés de la municipalité. Bonnes connaisseuses de la réalité des jardins, ces personnes sont responsables de la dynamisation, de l'articulation et de la cohésion du collectif. Leur rôle est central dans l'organisation, même s'il parait en même temps sujet à controverse puisqu'il crée la possibilité d'une promotion par le travail, engendrant un double risque de hiérarchisation et de clientélisme par rapport aux autres maraîchères et maraîchers. Puis de jeunes techniciens universitaires, dont une majorité (mais ils ne sont pas seuls) d'ingénieures et ingénieurs agronomes, formés à l'agroécologie et fortement motivés par le social et l'action solidaire, sont progressivement entrés dans le projet.

Figure 8 : Atelier participatif de l'équipe technique et des membres du PAU

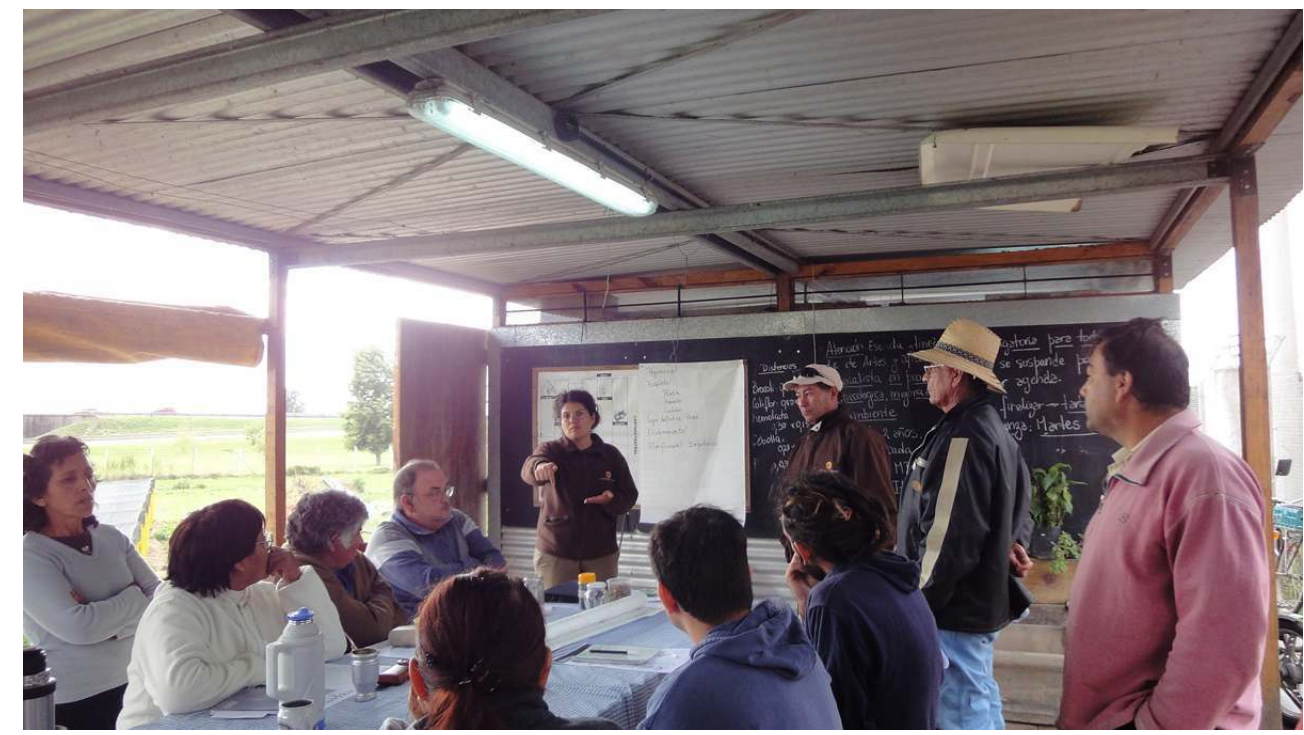

Cliché M. Soler Montiel

L'équipe technique du PAU dépend du Secrétariat de la promotion sociale, Soussecrétariat de l'Économie solidaire de la municipalité de Rosario, et elle est étroitement liée à l'Institut national des technologies agraires (INTA) à travers le programme ProHuerta ${ }^{17}$. Elle établit des contacts et négocie avec les autres départements de la municipalité, à la fois pour repérer les terrains disponibles et pour inclure l'agriculture urbaine dans les plans d'urbanisation.

Au plan local, le PAU maintient des relations solides et stables avec l'Université nationale de Rosario, à travers plusieurs facultés ${ }^{18}$ avec lesquelles ont été développés divers projets de soutien à la recherche et de renforcement du programme, faisant ainsi de l'agriculture urbaine un objet d'étude et une matière d'enseignement.

$\mathrm{Au}$ niveau national et international, le PAU a établi des liens avec des organismes de coopération internationale et monté des projets ${ }^{19}$. Et c'est avec l'aide de la Fondation $\mathrm{RUAF}^{20}$ que le PAU a assuré sa visibilité à l'échelle l'internationale, car elle lui a permis de s'insérer dans un important réseau d'agriculture urbaine où il a obtenu plusieurs prix. Le travail de diffusion et de recherche de visibilité développé par l'équipe technique a permis au PAU d'être considéré aujourd'hui comme l'une des références mondiales des expériences de politique publique en matière d'agriculture urbaine (FAO 2014). 


\section{La dimension citoyenne}

25 Le PAU a recherché activement, et par diverses voies, la reconnaissance du public et le soutien citoyen ${ }^{21}$. Depuis ses débuts, le principal moyen d'établir du lien social a été l'achat-vente de ses aliments et produits, à l'occasion d'événements hebdomadaires en place publique, donnant la possibilité aux jardinières et jardiniers d'entrer directement en contact avec la population de la ville. Dans le cas des paniers, les consommateurs établissent une relation directe plus stable à travers les demandes et la distribution à domicile, créant ainsi un lien de confiance et un engagement plus solide que celui qui prévaut dans l'achat sur les marchés publics (Mazzuca et al. 2009).

Le Programme a reçu des appuis de diverses organisations citoyennes de la ville, et il a développé des activités avec elles, en particulier le Réseau vie verte, qui a fonctionné entre 2008 et 2012 dans le but de faire émerger la consommation responsable. Il a centré une partie de ses activités sur la structuration d'un réseau de consommateurs stable, quelque 450 personnes, pour favoriser le commerce équitable des productions locales (FAO 2014). Après la dissolution du Réseau vie verte en tant que tel, une partie de ses membres ont créé un regroupement de consommateurs agroécologiques locaux à Rosario, la Compagnie naturelle ${ }^{22}$, qui se fournit en particulier auprès du réseau des jardiniers du PAU.

27 Un autre lien important s'est noué avec la population de Rosario par le biais des activités d'éducation pour l'environnement, et ces dernières années, avec les écoles en particulier. Le PAU s'est montré très actif dans l'organisation d'actions éducatives s'adressant au public en général dans le but de promouvoir une pratique alimentaire responsable en rapport avec l'agriculture urbaine. Soutenue par des cours portant sur le potager familial comme un passe-temps bon pour la santé, cette activité éducative a pris de l'ampleur, mobilisant surtout une population de classe moyenne et moyenne-supérieure. Des organisations sociales de plusieurs jardins en ont résulté, dans des espaces publics dispersés, comme stratégie éducative et de promotion d'une vie saine.

Ce vaste réseau de relations entre les trois espaces sociaux identifiés a conféré un caractère distinctif au PAU.

\section{Une multifonctionnalité difficile à gérer}

Le Programme n'a cessé d'amplifier ses activités et ses relations, pour répondre aux besoins internes de consolidation de l'activité agricole, aux demandes sociales et aux pressions institutionnelles. Nous allons analyser les principaux secteurs développés en identifiant les résultats obtenus et les limites, les contradictions et les défis lancés. On prêtera une attention toute particulière à la création d'emplois et de revenus autour de la filière agroalimentaire locale parce qu'elle a constitué l'objectif prioritaire au début, et qu'elle continue de représenter l'activité principale.

\section{L'agriculture urbaine génératrice d'emplois et de revenus}

Le PAU a ainsi créé une nouvelle filière agroalimentaire urbaine à Rosario, qui s'efforce d'intégrer une population urbaine d'exclus en articulant production locale de légumes frais et consommation citadine. Il est parvenu à créer son propre système de contrôle de 
qualité, avec un logo distinctif, qui s'appuie sur une surveillance et un conseil technique sur le terrain, des analyses en laboratoire et la confiance de ceux qui mangent ces aliments.

En 2013, les ventes ont atteint environ cent tonnes de légumes et cinq tonnes de plantes aromatiques et médicinales (FAO 2014). Il est cependant difficile d'évaluer les revenus générés, à cause, entre autres, de la diversité des situations personnelles et familiales des maraîchers. Les évaluations monétaires exigent un effort constant et systématique d'enregistrement de l'information qui requiert du personnel technique dédié exclusivement à cette tâche, ce qui n'a pas été possible. D'autre part, les personnes qui cultivent les parcelles n'ont pas l'habitude de quantifier et quelques-unes n'ont pas les moyens d'enregistrer l'information relative aux quantités produites et vendues, et aux revenus dégagés. À cela s'ajoute la nécessité d'évaluer le coût des matériels utilisés et du travail fourni par la ville et le PAU, ce qui est toujours difficile. Même si des tentatives ont été faites pour produire ponctuellement ces estimations, on peut dire des résultats qu'ils ont été partiels, et fort peu représentatifs. Il est clair, cependant, que l'agriculture urbaine offre à un ensemble de familles leur principal revenu stable, et un véritable mode de vie. Pour l'un des groupes familiaux, l'agriculture urbaine engendre des revenus complémentaires essentiels à leur survie ${ }^{23}$. Dans ces conditions, un des succès majeurs du PAU a été d'avoir consolidé une activité capable de générer des revenus significatifs dans la ville pour une population marginalisée, rencontrant des difficultés structurelles dans la quête du travail. Mais l'agriculture urbaine à Rosario n'est en réalité pas perçue comme une source d'emploi stable et suffisamment rémunératrice, ce qui constitue une contradiction de poids pour le Programme. Nous allons creuser cette question.

Nous avons vu que les réussites obtenues dans la création des emplois et des revenus sont le fruit d'un processus complexe et parfois contradictoire, porteur également d'apprentissages remarquables, soulevant des interrogations sur les limites et les défis du futur. Dans le PAU, la création d'emplois et de revenus est conditionnée par des facteurs externes et internes.

\section{Les facteurs externes}

La plupart des terres qui ont été attribuées au Programme étaient des espaces urbains vacants dépourvus de tout usage alternatif et elles étaient de mauvaise qualité agronomique. Même si certaines techniques agroécologiques permettent de rétablir la fertilité du sol au prix d'un travail continu et prolongé, la productivité reste modeste. Sur les sols contaminés impropres à la culture des aliments, des cultures florales ont été installées, ce qui permet de compléter l'offre du PAU sur les marchés. Même dans ces conditions, le processus de recherche, de négociation et de légalisation de l'accès à la terre a été particulièrement long et onéreux (Mazzuca et al. 2009, Lattuca 2006). Il a fallu également, pour obtenir l'homologation et le visa sanitaire des agro-industries, se lancer dans une bataille institutionnelle et légale compliquée contre une législation élaborée pour les grandes industries. Elle bloque les nouveaux producteurs artisanaux, surtout en matière de cosmétique naturelle.

L'agriculture pratiquée dans le cadre du PAU est exclusivement intra-urbaine ${ }^{24}$ puisqu'il n'a pas eu accès à la ceinture maraîchère et fruitière, bénéficiant de terres riches et intégrée au système de l'agriculture conventionnelle ${ }^{25}$. Elle bénéficie des techniques 
mécanisées et s'insèrent dans un réseau d'infrastructures publiques comprenant deux marchés de gros, fournissant la ville en aliments à travers des canaux de commercialisation dominants. Ces terres agricoles ont une haute valeur sur le marché et restent inaccessibles à la population pauvre et exclue avec laquelle travaille le PAU.

Par ailleurs, les bas prix des aliments (donnée structurelle du secteur agricole), et par conséquent les revenus réduits tirés des ventes, sont un autre facteur limitant extérieur. Les cultivateurs recherchent donc des moyens complémentaires, ce qui réduit d'autant le temps consacré au jardin, et donc la capacité de produire et de générer des recettes. La volonté du PAU de transformer l'agriculture urbaine en emploi à temps plein permettant de nourrir dignement toute une famille trouve là ses limites. Bien plus, les caractéristiques spécifiques du marché du travail argentin, et particulièrement rosarien, marqué par un chômage de courte durée, induisent de fréquents changements d'emplois et une alternance soutenue entre périodes d'activité courtes et chômage (Sánchez Miñaro 2014). Ceci se traduit par une rotation élevée au sein du collectif des jardiniers. L'agriculture ne peut être autre chose qu'un complément de revenu, fondamental dans la stratégie des familles pauvres, mais insuffisant à lui seul. L'instabilité est chronique et la possibilité d'incorporer de nouvelles personnes est faible.

prix des légumes sur les marchés sont fixés chaque semaine, en prenant comme référence les prix moyens affichés dans un échantillon d'épiceries locales, relevés par l'équipe technique. On essaye de ne pas réclamer des prix trop élevés afin de rendre les produits accessibles à la majorité de la population, même s'il faut reconnaître que la plus grande partie des consommateurs du PAU appartient à la classe moyenne supérieure des quartiers du centre de la ville, où se tiennent les marchés. L'éventualité d'augmenter les prix dans le but d'accroître les revenus des jardiniers, avec le risque de favoriser l'élitisme ou le manque de solidarité, fait l'objet d'un vif débat dans le programme.

La demande alimentaire réclame de la stabilité et de la diversité dans l'approvisionnement des produits, sous peine de voir déserter le consommateur, ce qui est difficile pour différentes raisons. Raisons climatiques conjoncturelles, et raisons structurelles on l'a vu, avec le manque de terres et leur faible qualité.

Parmi les facteurs externes il faut prendre en compte les valeurs partagées de façon inconsciente par l'ensemble de la société. La culture urbaine est défavorable à l'agriculture, perçue comme une affaire de pauvres et assimilée pour beaucoup à un échec social, surtout pour ceux qui ont émigré vers la ville en quête d'insertion. Elle n'est pas reconnue comme une activité digne ni souhaitable, et le défi majeur du PAU a consisté à donner une identité positive à l'agriculture urbaine. L'objectif était qu'elle soit assumée avec fierté et dignité, tout en préservant les origines indiennes guarani et toba, et en suivant les principes de l'agroécologie.

L'autre facteur culturel qui touche le PAU concerne le régime et les habitudes alimentaires. La consommation des protéines animales, et en particulier de la viande, est au centre de la culture argentine. La grillade de bœuf est le plat national des jours de fêtes et de célébrations. Perçus comme une nourriture de pauvres, les légumes occupent une place limitée dans le régime alimentaire et ils ne participent ni des choix gustatifs ni de la gastronomie. Ceci concerne tout à la fois les jardiniers (c'est un frein à leur capacité d'autoconsommation) et le consommateur urbain en général (cela freine les ventes du PAU). L'équipe technique s'efforce de changer les habitudes alimentaires des jardiniers et diverses opérations de promotion et d'éducation pour une alimentation saine visent la population en général. 


\section{Les facteurs internes}

Nous avons vus que les difficultés à garantir une offre suffisante en quantité et diversité de produits frais durant l'année entière sur les places de marché et pour les paniers constituent une des faiblesses internes majeures du PAU. Elles résultent autant de facteurs externes évoqués plus haut, que de déficiences internes bien réelles. De nombreux jardiniers maîtrisent mal les techniques agricoles, car ils ont vécu la plus grande partie de leur vie déconnectés des cycles de la nature et de l'agriculture. La plus grande partie d'entre eux ne dispose d'aucune connaissance empirique paysanne, ou elle est pour le moins érodée. L'apprentissage des pratiques agroécologiques est fondamental pour le PAU, mais le turn-over réduit l'efficacité de la formation.

41 L'hétérogénéité socioculturelle entre également en jeu et dans ce contexte ${ }^{26}$, les parcours individuels et sociaux des cultivateurs deviennent un facteur central. Les personnes qui s'insèrent le mieux dans le Programme sont celles qui disposent de réseaux familiaux structurés et stables. Dans plusieurs cas, l'agriculture fait partie d'une stratégie familiale avec une division du travail entre hommes et femmes, et aussi entre adultes et jeunes. Ainsi la famille tire-t-elle les meilleurs revenus possibles de l'agriculture, en les combinant avec d'autres travaux ponctuels ou à temps partiel pour compléter les rentrées d'argent. Mais ceux qui sont le mieux insérés dans de solides réseaux familiaux ou sociaux sont à même d'obtenir un emploi alternatif, et ils ont peu de raisons de se rapprocher, ou d'appartenir au PAU. Une bonne partie des jardiniers du PAU éprouvent de grandes difficultés à prendre en mains la planification des cultures, assumer le travail dans les jardins et la commercialisation de leurs produits par les divers moyens mis à leur disposition. Ils manquent de formation et dans leur état de pauvreté chronique, ils sont fragiles sur le plan psycho-émotionnel. Développer les capacités personnelles d'organisation, de gestion et de commercialisation chez les jardiniers est aussi important que de leur apprendre à cultiver avec des techniques agroécologiques.

Cette population en situation de pauvreté extrême ne dispose d'aucune capacité d'investir ni d'assumer les risques économiques. En découle une dépendance économique vis-à-vis des intrants, des infrastructures et des services que l'équipe technique, les réseaux institutionnels et les réseaux citoyens fournissent à la chaîne agroalimentaire du PAU. Cette vulnérabilité économique devient dramatique lorsque les conditions climatiques sont mauvaises et que la récolte est perdue. Il n'existe pas à ce jour de système de couverture efficace.

À cette dépendance économique et matérielle s'ajoute la dépendance en ce qui concerne l'organisation pour planifier et conduire le jardinage, les agro-industries et surtout, la commercialisation. L'installation et le « démontage » des parcelles et du matériel (tunnels et serres) est à la charge de la municipalité, l'équipe technique aide pour le transport, des lieux de production aux marchés, et pour composer les paniers. Leur distribution est restée aux mains de l'équipe technique pendant la longue étape initiale. C'est là sans doute la principale faiblesse interne au PAU et l'objet d'un des défis majeurs pour l'avenir. 
Figure 9 : Construction d'un abri ombragé dans le jardin du PAU du Hogar Español

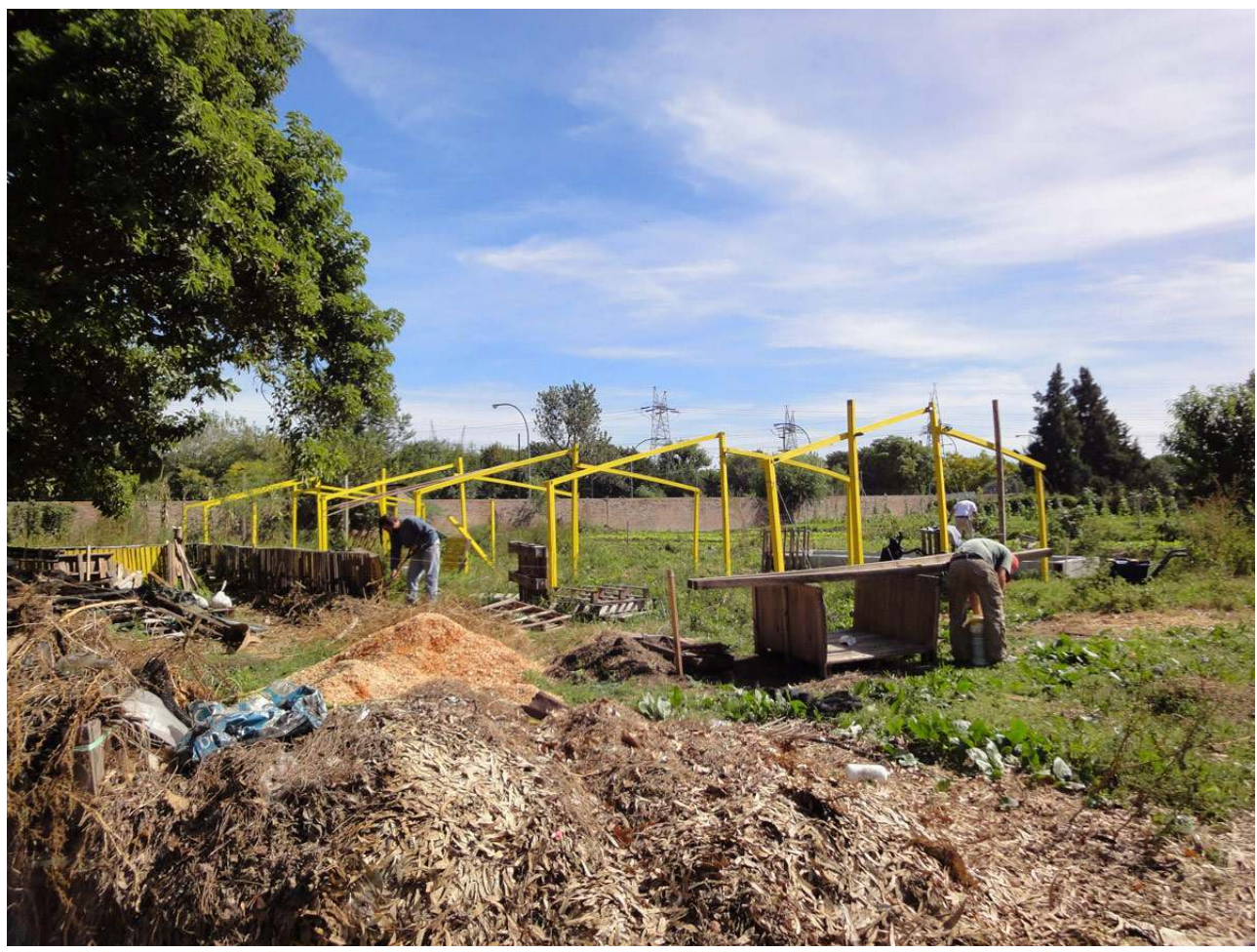

(C) Programa de Agricultura Urbana de Rosario, Argentina

\section{L'agriculture urbaine, l'insertion et la recherche de cohésion sociale}

Le PAU cherche à promouvoir l'intégration et la cohésion sociale, établir un courant de confiance à mesure que se renforce l'appartenance à un groupe. Le fait de cultiver dans des jardins communautaires où chacun devient responsable d'une parcelle tout en participant à un projet commun crée un espace pour l'apprentissage des valeurs de solidarité et de soutien mutuel. En favorisant les aptitudes personnelles à gérer son travail dans le temps, on stimule la motivation, le sens des responsabilités et l'autonomie. Il en résulte une meilleure estime de soi, de plus grandes facultés à aller vers les autres, prendre des initiatives et se prendre soi-même en charge. Tels étaient les objectifs du PAU, dès ses débuts (Lattuca 2006, Mazzuca et al. 2009) même si un doute persiste sur le type d'orientation à privilégier dans le conseil et l'appui technique : la productivité ou le social.

45 La complexité et l'hétérogénéité sociale du collectif maraîcher se retrouve dans une diversité des profils sociaux. Ceci implique de démultiplier les objectifs et les programmes et de faire intervenir différentes équipes techniques. Une politique de développement agricole requiert une équipe formée au technico-productif en même temps qu'à l'agroécologie sociale, tandis qu'une politique d'insertion sociale doit mobiliser une équipe technique de psychologues et de pédagogues tournée vers la dynamisation sociale et, parfois même, vers le soutien psychologique, social et sanitaire. 

vulnérables, trop dépourvus sur le plan familial et personnel, ont du mal à s'engager sur le long terme, dans un projet de production rémunérateur et stable. Ils bénéficient d'un apprentissage social, ils vont mieux sur le plan personnel et leurs chances de prendre pied dans le marché du travail augmentent. Mais il y a une bonne part de supputation dans cette évaluation des succès obtenus par le programme en termes d'insertion sociale par le travail bilan, car il est difficile de suivre les itinéraires professionnels et personnels de ceux qui abandonnent le Programme. Ne pourrait-on pas considérer que l'important turn-over des usagers du PAU constitue un signe de réussite de la politique d'insertion, plutôt que la preuve de son échec?

où l'objectif prioritaire clairement annoncé est la promotion de l'emploi en agriculture urbaine, le succès est avant tout mesuré, tant par l'équipe technique et par les administrations publiques, en termes de participants stables et de revenus moyens annuels. Il faudrait donc se doter d'indicateurs complémentaires dans les domaines du social et de l'environnement. adapté aux objectifs prédéfinis par le programme. Car dans la pratique, l'équipe technique ne parvient pas à sélectionner le collectif des usagers et elle se voit contrainte de travailler avec une population hétérogène, dont une majorité de femmes chargées de famille empêchées de se consacrer à plein temps à la production et à la vente de produits frais. Cette difficile réalité socioculturelle du groupe des jardiniers n'est en fait pas acceptée, au moins par une partie de l'équipe technique, démunie devant les besoins de ces personnes.

la très forte hétérogénéité des profils sociaux impliquerait qu'on s'engage à définir des politiques d'intervention alternatives en matière d'agriculture urbaine. Il serait même nécessaire de fixer des objectifs et des indicateurs adaptés aux divers profils identifiés afin qu'on en puisse évaluer l'efficacité. Il s'agit là d'un autre défi organisationnel interne, un défi fondamental.

\section{L'agriculture urbaine et les politiques municipales de la ville durable}

La ville s'est construite en opposition à la campagne, et l'agriculture n'y trouve pas véritablement sa place (Verdaguer 2014). Vouloir la réincorporer au titre de la ville durable est un grand défi. C'est pourquoi, le PAU a cherché à convaincre, dès l'origine, des interlocuteurs dans les divers départements de la municipalité et de l'État. Des résultats ont été obtenus, comme l'incorporation de l'agriculture urbaine dans le Plan d'Organisation Territoriale 2007-2017 et dans le Plan Métropolitain Stratégique 2008-2018 (FAO 2014).

51 La suspension précipitée, en 1997, du programme de jardins collectifs de la municipalité a fait prendre conscience de la nécessité d'introduire des objectifs et des rythmes politiques dans la planification du PAU. Si elle veut survivre, une institution doit présenter aux citoyens des résultats quantifiables. En ce sens, et ces dernières années davantage encore, une part importante de l'activité a consisté à rendre visibles les « externalités " positives des parcs-jardins potagers, espaces de production et de détente créés sur des dépotoirs, des friches industrielles ou des terrains inondables laissés à l'abandon. L'amélioration du

Revue d'ethnoécologie, 8 | 2015 
paysage, la contribution à la gestion des déchets urbains, l'augmentation de l'offre de zones vertes et récréatives de la ville, et la réduction des coûts dans l'acquisition et la préservation de ces espaces publics constituent des services environnementaux, représentent des bénéfices sociaux et économiques, hautement valorisés dans un monde néo-libéral cherchant à réduire les dépenses liées à l'action publique locale (Rosol 2010). On peut ajouter le rôle bénéfique de l'agriculture urbaine sur le réchauffement climatique, récemment reconnu (De Zeeuw 2011).

Figure 10 : Parc-jardin de La Tablada : affichage du multi partenariat

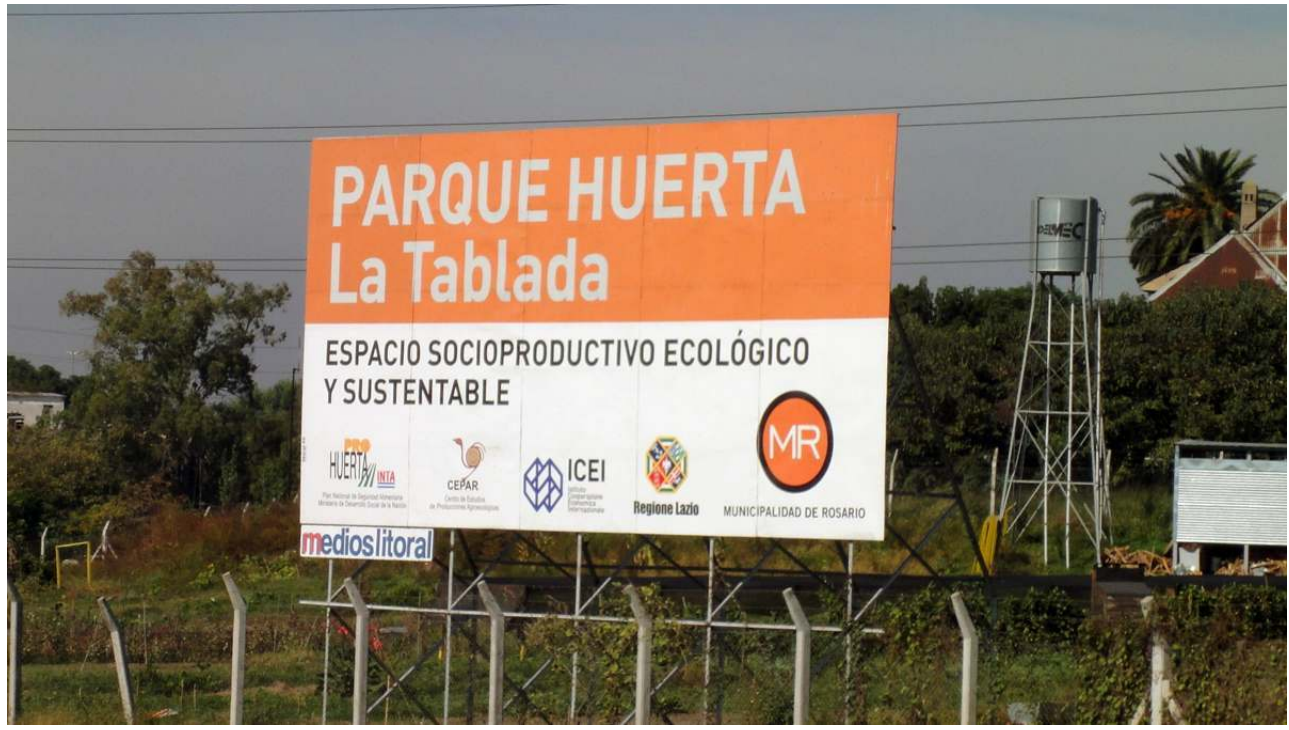

Cliché M. Soler Montiel l'agriculture urbaine, et développe toujours plus d'activités nouvelles ${ }^{27}$, en prêtant une attention particulière aux services environnementaux. Il arrive qu'on s'éloigne ainsi des besoins de la population pauvre chargée de cultiver les jardins (c'est le cas de la question $\mathrm{du}$ ralentissement du réchauffement climatique). Mais ces thèmes sont prioritaires pour les administrations publiques et les organisations de coopération internationale au développement, et ils prennent une place grandissante dans les agendas politiques écologiques internationaux.

On risque d'arriver ainsi à une situation paradoxale, voire contradictoire. L'amélioration de la visibilité et de l'impact politique pourrait accaparer les ressources humaines disponibles, au détriment du temps et de l'attention portés au collectif des maraîchers. Prévenir une telle situation impose une organisation attentive et une distribution rigoureuse des tâches au sein de l'équipe technique.

\section{Éducation à l'environnement, loisirs et participation citoyenne}

Depuis ses débuts, le PAU n'a cessé d'organiser des activités d'éducation à l'environnement destinées à toutes sortes de collectifs. La formation et la qualification du collectif maraîcher font toujours l'objet d'une attention particulière. Une vingtaine de maraîchers ont reçu en 2013 une certification du ministère du Travail, de l'Emploi et de la 
Sécurité sociale, en tant que spécialistes en cultures écologiques. Cette distinction a été obtenue grâce au programme de formation conçu par l'équipe technique du PAU, qui travaille à présent sur la question de l'insertion de jeunes chômeurs (18-25 ans), qui se voient attribuer des parcelles de culture (FAO 2014).

$\mathrm{Au}$ cours des dernières années, les activités d'éducation à l'environnement destinées aux enfants des écoles de la ville ont repris, avec un propos plus ludique que celui du Réseau d'enseignants jardiniers des étapes initiales. Elles s'ouvrent à l'ensemble des citadins tout au long de l'année, mais plus particulièrement en septembre, à l'occasion de la célébration de la semaine de l'agriculture urbaine.

Dès 2005, les parcs-jardins ont accueilli des activités sportives et de loisirs, pour les citadins en général.

Mais elles répondent en priorité aux besoins des classes moyennes et moyennessupérieures $^{28}$, et on s'est éloigné des motivations initiales, centrées sur la population les pauvres et les exclus. Elles contribuent néanmoins à casser les codes de la discrimination sociale, parce qu'elles construisent une image favorable du secteur de l'agriculture urbaine. Il y aurait donc comme un défi supplémentaire à vouloir faire partager de tels espaces, sans tomber dans les conflits d'usage entre des groupes motivés par des besoins et des priorités différents.

\section{Les défis pour le futur}

58 Le PAU avait été conçu pour créer des emplois et des revenus, par le truchement de l'agriculture urbaine et pour la population pauvre. L'objectif majeur était l'insertion sociale par le travail, en rupture avec l'assistanat habituel des autres politiques de lutte contre la pauvreté. Une chaîne agroalimentaire locale a donc été construite. Aujourd'hui, elle relie un réseau de jardins agroécologiques, deux agro-industries artisanales et un réseau local de consommation articulé autour des marchés, d'une vente de « paniers » et de groupements de consommateurs.

Tout au long de ces années, le PAU a développé des activités en intégrant de nouveaux acteurs. Cette diversification répondait aux exigences de l'administration publique locale. Elle résultait aussi des propositions émanant des universités, des ONG et des organismes internationaux, et des initiatives de l'équipe technique. Le PAU est aujourd'hui l'une des rares expériences pratiques de la multifonctionnalité, caractéristique de l'agriculture urbaine.

60 La recherche a montré que la création d'emplois stables et de revenus suffisants se heurte principalement à des facteurs externes difficilement contrôlables par l'équipe. Si elle a les moyens d'intervenir sur la capacité des jardiniers à s'organiser et à produire, permettre à ces derniers et plus largement à l'ensemble des jardins et des agro-industries d'acquérir une certaine autonomie reste un défi majeur: faire en sorte que les jardiniers soient capables de provoquer et d'animer leurs propres réunions, de planifier la production et la commercialisation, de vivre de leur travail reste aujourd'hui un problème sans solution.

61 L'hétérogénéité sociale et l'extrême précarité des personnes invitent à considérer le PAU avant tout comme un programme social de lutte contre la pauvreté et l'exclusion, à accorder aux objectifs sociaux la même importance qu'à l'insertion professionnelle, ce qui implique de développer des politiques adaptées à la diversité des situations de vulnérabilité. 
62 On sait à présent que les objectifs de la multifonctionnalité ne sont pas toujours complémentaires. Les besoins des jardiniers en matière d'assistance technique et le renforcement de l'organisation collective ne sont pas toujours compatibles avec les exigences de l'agenda politique des administrations publiques, des gouvernements locaux, régionaux et nationaux, ou des institutions et ONG internationales ni avec les envies de détente et de distraction des classes moyennes. Le manque de moyens, au regard des ambitions affichées de la multifonctionnalité, impose de hiérarchiser les objectifs et de définir des priorités, d'autant plus que la place de l'agriculture urbaine dans les villes « durables » peine à être reconnue.

Il importe de définir les objectifs prioritaires d'une politique d'agriculture urbaine. Le PAU peut constituer un cas d'école.

L'auteur remercie Marta Sáchez Miñarro pour ses suggestions et corrections qui ont substantiellement contribué à l'analyse.

\section{BIBLIOGRAPHIE}

Altieri M.A. 1987 - Agroecology: the Scientific Basis of Alternative Agriculture. Boulder Colorado, Westview Press, 227 p.

Urban agriculture : findings from four city case studies.

Banque Mondiale 2013 - Urban agriculture: findings from four city case studies. Urban development series ; knowledge papers no. 18. Washington DC : World Bank. http://

documents.worldbank.org/curated/en/2013/07/18165126/urban-agriculture-findings-four-citycase-studies. Consulté le 28/07/2014.

Bellows A. C., Brown K. \& Smit J. 2005 - Health Benefits of Urban Agriculture. A paper from the Community Food Security Coalition's North American Institute of Urban Agriculture, Venice, CA. http:foodsecurity.org.pubs.html. Consulté le 28 juillet 2014.

CBD 2012 - Cities and biodiversity Outlook: Action and Policy. Montreal, Secretariat on Biological Diversity. http://www.cbd.int/doc/publications/cbo-booklet-2012-en.pdf. Consulté le 28/07/2014.

Clarín 2014 - Una familia necesitó \$ 5.366 en septiembre para no caer en la pobreza. http:// www.ieco.clarin.com/economia/FIEL-pobreza-septiembre-CBA-CBT_0_1226877810.html. Consulté le 24/11/2014.

De Zeeuw H. 2011 - Cities, Climate Change and Urban Agriculture Urban. Agriculture Magazine 25 : $39-42$.

FAO 2014 - Ciudades más verdes en América Latina y el Caribe. Un informe de la FAO sobre la agricultura urbana y periurbana en la región. http://www.fao.org/ag/agp/greenercities/pdf/GGCLAC/ Ciudades-mas-verdes-America-Latina-Caribe.pdf. Consulté le 28/07/2014.

Gliessman S.R. 1989 - Agroecology. Researching the Ecological Basis for Sustainable Agriculture. New York, Springer-Verlag. 
INDEC 2010 - Censo Nacional de Población, Hogares y Vivienda 2010, Instituto Nacional de Estadísticas y Censos, Argentina. http://www.censo2010.indec.gov.ar/ Consulté le 24/11/2014.

La Nación 2014 - El gobierno acordó una suba de 31\% para el salario mínimo. http:// www.lanacion.com.ar/m1/1723724-el-gobierno-acordo-una-suba-de-31-para-el-salario-minimo. Consulté le 24/11/2014.

Largo-Wight E. 2011 - Cultivating healthy places and communities: evidenced-based nature contact recommendations. International Journal of Environmental Health Research 21 (1) : 41-61.

Lattuca A. 2006 - Parques huerta : una estrategia de uso del suelo para la agricultura urbana en la ciudad de Rosario, Provincia de Santa Fe, Argentina. Tesina de Maestría en Agroecología, Universidad Internacional de Andalucía (UNIA), Baeza, España.

Mazzuca A., Ponce M. \& Terrile R. 2009 - La agricultura urbana en Rosario: balances y perspectivas. Lima, IPES, $182 \mathrm{p}$.

Mougeot L. J. 2000 - Urban agriculture: definition, presence, potentials and risks. In Bakker N., Dubbeling M., Guendel S., Sabel Koshella U. \& Zeeuw de H. (Ed.) Growing cities, growing food. DSE, Felfafing Germnay : 1-42.

Oudwater N., de Vries M., Renting H. \& Dubbleling M. 2013 - Thematic paper 2: Innovative experiences with short food supply chains in (peri-)urban agriculture in the global South. ETC Foundation and RUAF, Foundation www.ruaf.org/. Consulté le 28/07/2014.

Pérez-Vitoria S. 2005 - Les paysans sont de retour. Arles, Actes Sud, 272 p. (Questions de société). Renting H., Naneix C., Dubbeling M., Cai J. 2013 - Innovative experiences with multifunctional urban and peri-urban agriculture in city regions in the global South. Thematic paper 3, 73 p. www.ruaf.org/. Consulté le 28/07/2014.

Rosol M. 2010 - Public Participation in Post-Fordist Urban Green Space Governance: The Case of Community Gardens in Berlin. International Journal of Urban and Regional Research 34 (3) : 548-563.

Sánchez Miñarro M. 2014 - Agroecología urbana en la ciudad de Rosario (Argentina). Un breve análisis a una propuesta de gran complejidad. PAPELES de Relaciones Ecosociales y Cambio Global noำ124. Ed. Fundación FUHEM.

Sevilla Guzmán E. 2006 - Desde el pensamiento social agrario : perspectivas agroecológicas del Instituto de sociología y estudios campesinos. Córdoba, Servicio de Publicaciones de la Universidad de España.

Toledo V.M. 1993 - La racionalidad ecológica de la producción campesina. In : Sevilla Guzmán E. y González de Molina M. (Ed.) Ecología, Campesinado e Historia : 197-218.

UCA 2014 - Estimaciones de tasas de indigencia y pobreza (2010-2013) Totales Urbanos. Observatorio de la Deuda Social Argentina, Universidad Católica Argentina. http:// www.uca.edu.ar/uca/common/grupo68/files/ INDIGENCIA_Y_POBREZA_INFORME_PRENSA_abril_2014.pdf. Consulté le 24/11/2014.

Van der Ploeg J.D. 2008 - The New Peasantries. London, Earthscan.

Verdaguer Viana-Cárdenas C. (sous presse) - El campo y la ciudad, áreas de reencuentro. Hacia una Nueva Cultura del Territorio. Hábitat y Sociedad 6.

Zasada I. 2011 - Multifunctional peri-urban agriculture. A review of social demands and the $p$ rovision of goods and services by farming. Land Use Policy 28 : 639-648. 


\section{NOTES}

1. Debilidades, Amenazas, Fuerzas y Oportunidades (Faiblesses, Menaces, Forces et Opportunités). 2. En 2013, d'après l'Observatoire de la dette argentine de l'Université catholique argentine, le taux de pauvreté dans les grandes agglomérations du pays était estimé à $25,6 \%$ selon certains analystes et à $27,5 \%$ selon d'autres, et le taux d'indigence, compris entre 5,1\% et 5,5\%. En 2002, ces taux atteignaient respectivement $54 \%$ et $27,7 \%$.

3. Des habitants d'un bidonville situé dans le sud de Rosario, près du ruisseau Saladillo, se regroupent pour obtenir l'accès à des logements stables et pour organiser l'approvisionnement en biens de première nécessité. C'est dans cette coopérative, créée par une communauté pauvre, qu'une première alliance se noue avec un groupe d'ingénieurs agronomes.

4. Une grande partie de l'équipe technique du PAU s'est formée à l'ISEC (masters et thèses de doctorat en agroécologie).

5. Le ministère de l'Agriculture, de l'élevage, de l'industrie et du commerce de la province de Santa Fe (MAGIC), l'Institut national de technologies agricoles (INTA), la Faculté de Sciences agraires de l'Université nationale de Rosario et un réseau d'ONG, comme le Centre d'études et de technologies appropriées pour l'Argentine (CETAAR), le Centre de communication populaire et de conseil légal (CECOPAL), le Centre d'études et d'action environnementales (CEAA) de Rosario, et le Centre d'éducation et technologie du Chili (Lattuca 2006, Mazzuca et al. 2009).

6. D'après les données de l'équipe technique du PAU, le programme a permis de développer de nombreux jardins familiaux, communautaires et scolaires et la mise place d'un réseau de maraîchers et maraîchères ainsi qu'un réseau d'apprentis maraîchers (Rateau, pelle et envies) intégrant plus de deux cents élèves de maternelles et d'écoles primaires (une centaine d'écoles de la région). Près de trois cents jardins scolaires et familiaux ont été mis en culture (Mazzuca et al. 2009).

7. Des lieux de transformation artisanale et à petite échelle de légumes, plantes aromatiques et cosmétiques ont été appelés «agro-industries", pour rendre visible l'effort réalisé de transformation et de création de valeur ajoutée.

8. L'ONG italienne Instituto de Cooperazione Economica Internazionale (ICEI) met en effet en place un Projet de renforcement du Programme d'Agriculture Urbaine de Rosario, financé par la Coopération internationale italienne (1,5 million d'euros). Il fonctionne entre 2008 et 2010.

9. En 2011 ce réseau est devenu une association sans but lucratif.

10. Le parque-huerta est un espace public, ouvert à l'ensemble des habitants, spécialement aménagé pour pouvoir combiner agriculture urbaine et activités éducatives et de loisirs. Ces espaces s'inspirent de l'expérience des potagers écologiques d'auto-consommation de Miraflores à Séville, en Andalousie, situés dans un parc public.

11. Il s'agit des maraîchers de la première étape du PAU qui ont fini par contractualiser avec la municipalité pour participer à la dynamisation et à l'articulation sociale dans les potagers du programme.

12. Aujourd'hui, l'occupation des sols est majoritairement légalisée et stable, aboutissement d'un long processus, initié par l'équipe technique, d'identification des espaces disponibles non marchandisables et de négociations avec les institutions publiques.

13. Hydroponie biologique.

14. Afin d'assurer la stabilité de l'occupation des sols dès le début, le PAU a privilégié les espaces publics non constructibles, délaissant les sols privés qui ont été progressivement urbanisés.

15. L'origine du potager est en fait antérieure, liée à l'activisme des habitants pendant la crise de 2001, qui ont transformé le dépôt d'ordures d'un bidonville en terre agricole ouverte, transformée par la suite en parc-jardin. 
16. 500 pesos équivalent à $47-50$ euros, soit $11,4 \%$ du salaire minimum qui était en septembre 2014 de 4400 pesos.

17. Créé dans les années 1990, le Programme national Pro-Huerta soutient, dans l'ensemble du pays, l'agriculture urbaine familiale en vue de l'autoconsommation dans des jardins collectifs par l'apport de semences, de produits de base, et d'assistance technique. Une partie de l'équipe technique du PAU sont des techniciens de l'INTA relevant du Pro-Huerta.

18. Facultés d'architecture, des sciences agraires, des sciences politiques et d'ingénierie.

19. Projet déjà cité plus haut, qui a été développé en association avec l'ONG italienne «Istituto Cooperazione Economica Internazionale » (ICEI).

20. La Fondation RUAF est une organisation sociale sans but lucratif, centrée sur la diffusion et la promotion de l'agriculture urbaine au niveau mondial. Au cours de presque deux décennies d'existence, elle a construit un réseau international d'échanges d'expériences, de visibilité et d'appui pour des projets d'agriculture urbaine et périurbaine. Pour plus d'informations, voir www.ruaf.org

21. Il est impossible de résumer ici tous les liens développés par le PAU dans les réseaux tout au long de son existence. Pour plus de détails sur ce point, voir : Ottmann 2005, Lattuca 2006, Mazzuca et al. 2009.

22. http://companianatural.blogspot.com.es/

23. Dans le cadre du projet coordonné par RUAF, « Pour intégrer l'agriculture urbaine dans les stratégies d'adaptation et/ou mitigation face au changement climatique dans les villes ", une étude a été réalisée en 2013 sur le Parc Huerta Molino blanco, qui portait sur les revenus des diverses familles. Pour celles qui tirent leur revenu principal de l'agriculture, il se monte à 6.000 et 10.000 pesos par mois, soit entre 570 et 950 euros. Celles qui en tirent un complément gagnent entre 3.000 et 5.000 pesos ( 285 et 475 euros) ; quant à celles qui la pratiquent occasionnellement en tant qu'activité sociale et/ou thérapeutique, le gain est en moyenne de 1.000 pesos (environ 95 euros). Il faut tenir compte $\mathrm{du}$ fait que la Fondation des Recherches économiques latinoaméricaines (FIES) estime que le revenu mensuel minimum pour ne pas dépasser le seuil de la pauvreté en Argentine doit s'élever à 5.366 pesos, le coût du panier alimentaire de base pour un foyer de quatre personnes dans la ville de Buenos Aires étant de 2.947 pesos (Clarín 2014). On peut aussi se référer au salaire minimum qui a été fixé en septembre 2014 à 4.400 pesos (La Nación 2014).

24. En avril 2014, un projet pilote s'est mis en place dans la périphérie de Rosario, mais nous n'avons pas travaillé dans cette zone jusqu'à présent.

25. Toutefois la pratique continue de l'agriculture intensive n'a pas manqué d'affecter la qualité des sols qui réclament un usage de plus en plus intensif de produits et d'intrants industriels pour maintenir les rendements, avec comme conséquences un accroissement des coûts et une détérioration de l'environnement.

26. Sans aucun doute la situation socioéconomique du pays est le facteur externe qui détermine l'hétérogénéité socioculturelle des personnes concernées par notre recherche. Cette hétérogénéité est tenue dans cette analyse pour un facteur interne alors qu'elle doit beaucoup aux conditions structurelles du pays.

27. Enregistrement et analyse de données, valorisation du travail des chercheurs et des techniciens, rédaction de rapports, participation à des projets de recherche et de coopération internationale... Il est vrai que ces activités ont permis d'obtenir des crédits supplémentaires.

28. Depuis 2013, des cours de formation au jardin familial, sur les balcons et sur les toits ont été organisés pour les classes moyenne et supérieure. D'autre part, durant la Semaine de la gastronomie du mois de mai, on donne des cours de cuisine dans les parcs-jardins, en association avec un groupement de chefs de Rosario. 


\section{RÉSUMÉS}

L'agriculture urbaine prétend répondre efficacement aux défis de la crise globale actuelle en apportant des emplois et des revenus à ceux qui travaillent la terre, des aliments sains et durables à ceux qui mangent les produits, et des services environnementaux à l'ensemble des citoyens. Dans la ville argentine de Rosario (province de Santa Fe), pour faire face à la crise de 2001, l'agriculture urbaine est passée du stade d'alternative agronomique et socioéconomique proposée par l'agroécologie à une activité majeure. Elle s'est finalement institutionnalisée comme politique municipale. Dans ce processus, les agents, les espaces et les activités concernés ainsi que l'organisation et les relations institutionnelles se sont complexifiés, surtout dans la dernière décennie. À l'échelle mondiale, le Programme d'Agriculture Urbaine de la ville de Rosario est un des rares exemples de gestion publique de la multifonctionnalité de cette activité. L'article présente le programme et apporte une réflexion sur les réussites, les limites et les défis de sa réalisation.

La agricultura urbana es una actividad multifuncional que ha mostrado una elevada capacidad para responder a los retos de la crisis global actual aportando empleo e ingresos a quienes trabajan la tierra, alimentos sanos y sostenibles a quienes comen sus productos, así como servicios ambientales y sociales a la ciudadanía en su conjunto. En la ciudad argentina de Rosario, en la provincia de Santa Fe, la agricultura urbana pasó de ser una alternativa agronómica y socioeconómica propuesta desde la agroecología a convertirse en una actividad masiva en respuesta a la crisis económica de 2001 para finalmente institucionalizarse como política municipal. En este proceso tanto los agentes implicados, los espacios y las actividades realizadas como la organización y las relaciones institucionales se han complejizado, sobre todo en la última década. En la actualidad, el Programa de Agricultura Urbana de la ciudad de Rosario es uno de los pocos casos prácticos de gestión pública de la multifuncionalidad de la agricultura urbana. En este artículo se presenta la experiencia de este programa y se reflexiona sobre los logros, límites y retos que implica la gestión pública de la multifuncionalidad de la agricultura urbana.

Urban agriculture is a multifunctional activity that has proved a high capacity to provide alternatives to global crisis generating employment and incomes to who works the land, sustainable and healthy food to who eats its products, as well as social and environmental services to citizenship. In the Argentinean city of Rosario, in the province of Santa Fe, urban agriculture has moved from being an agronomic and socioeconomic alternative proposed by Agroecology to a massive activity in reaction to economic crisis in 2001 to become an institutionalized public local policy at present. In the process, actors involved, spaces and places, activities, organization and institutional links has gain in complexity, mainly in the last decade. At present, the Urban Agriculture Program in the city of Rosario is one of the few real cases of public management of urban agriculture multifuncionality. This article presents this experience and reflect on achievements, limits and challenges of public management of urban agriculture multifuncionality. 
INDEX

Palabras claves : agricultura urbana, agroecología, multifuncionalidad, ciudades sostenibles, sostenibilidad urbana, políticas locales sostenibles

Index géographique : Argentine, Rosario

Mots-clés : agriculture urbaine, agroécologie, multifonctionnalité, ville durable, durabilité urbaine, politique locale durable

Keywords : urban agriculture, agroecology, multifunctionality, sustainable cities, urban sustainability, sustainable local policies

\section{AUTEUR}

\section{MARTA SOLER MONTIEL}

Enseignante-chercheuse

Département d'Économie appliquée II - Université de Séville, Espagne

msoler@us.es 